\title{
Total Synthesis and Chemoproteomics Connect Curcusone Diterpenes with Oncogenic Protein BRAT1
}

Chengsen Cui ${ }^{1} \dagger$, Brendan G. Dwyer ${ }^{2} \dagger$, Chang Liu ${ }^{1}$, Daniel Abegg ${ }^{2}$, Zhongjian Cai ${ }^{1}$, Dominic $\operatorname{Hoch}^{2}$, Xianglin Yin ${ }^{1}$, Nan Qiu $^{2}$, Jieqing Liu ${ }^{3}$, Alexander Adibekian ${ }^{2 *}$, Mingji Dai ${ }^{1 *}$

${ }^{1}$ Department of Chemistry and Center for Cancer Research, Purdue University, West Lafayette, IN 47907 , United States

${ }^{2}$ Department of Chemistry, The Scripps Research Institute, 130 Scripps Way, Jupiter, FL 33458, United States

${ }^{3}$ School of Medicine, Huaqiao University, Quanzhou 362021, P. R. China

Correspondence and requests for materials should be addressed to M. D. (email: mjdai@purdue.edu) and

\section{A. A. (aadibeki@scripps.edu)}

${ }^{\dagger}$ Contributed equally.

\begin{abstract}
Natural products are an indispensable source of lifesaving medicine, but natural product-based drug discovery often suffers from scarce natural supply and unknown mode of action. The study and development of anticancer curcusone diterpenes fall into such a dilemma. Meanwhile, many biologicallyvalidated disease targets are considered "undruggable" due to the lack of enzymatic activity and/or predicted small molecule binding sites. The oncogenic BRCA1-associated ATM activator 1 (BRAT1) belongs to such an "undruggable" category. Here, we report our synthetic and chemoproteomics studies of the curcusone diterpenes that culminate in an efficient total synthesis and the identification of BRAT1 as a cellular target. We demonstrate for the first time that BRAT1 can be inhibited by a small molecule (curcusone D), resulting in impaired DNA damage response, reduced cancer cell migration, potentiated activity of the DNA damaging drug etoposide, and other phenotypes similar to BRAT1 knockdown.
\end{abstract}


Natural products have been valuable sources and inspirations of lifesaving drug molecules ${ }^{1}$. Their accumulated evolutionary wisdom together with their structural novelty and diversity makes them unparalleled for novel therapeutic development. However, their natural scarcity, structural complexity, and unknown mode of action often hamper their further development in the drug discovery pipeline. Total synthesis is frequently employed to solve the material supply and chemical probe synthesis for comprehensive biological profiling and target identification ${ }^{2}$. Meanwhile, many biologically validated disease targets are considered as "undruggable" from a chemical standpoint due to the lack of enzymatic activity and/or small molecule binding sites ${ }^{3}$. The BRCA1-associated ATM activator 1 (BRAT1) protein has been validated as an oncogenic protein involved in various cancers but belongs to the "undruggable" category with no known small molecule inhibitors to date. Herein, we report a collaborative effort in the total synthesis and chemoproteomics profiling of curcusone natural products which reveals BRAT1 as a key cellular target and validated curcusone D as the first BRAT1 inhibitor.

The curcusone diterpenes (Fig. 1A) were isolated from Jatropha curcas, a widely used ingredient in traditional remedies for a variety of ailments including cancer. Structurally, they share a characteristic [6-7-5] tricyclic skeleton with the daphnane and tigliane diterpenes ${ }^{4}$. Curcusones A-D (1a-d), isolated by Clardy and co-workers in 1986, were unambiguously identified as two epimeric pairs at the C2 position ${ }^{5}$. Since then, around thirty curcusone molecules have been isolated including curcusones F- $\mathrm{J}^{6}$, which lack the dienone moiety in the seven-membered ring. Structurally rearranged analogs like spirocurcasone (3) and dimeric analogs such as dimericursone A (2a) and dimericursone B (2b) were discovered recently ${ }^{7,8}$. Among them, 1a-1d exhibited low micromolar $\mathrm{IC}_{50}$ values against a broad spectrum of human cancer cell lines $^{6}$. However, their mode of action remained unknown and no total syntheses of 1a-1d were reported prior to this study. 


\section{A Curcusone Natural Products}

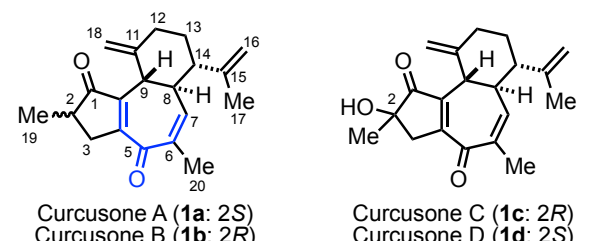

Curcusone $\mathrm{B}(1 \mathrm{~b}: 2 R) \quad$ Curcusone $\mathrm{D}(1 \mathrm{~d}: 2 S)$

anti cancer cell proliferation and antimetastatic

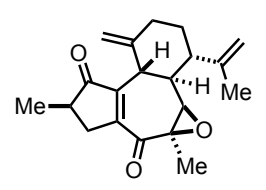

Curcusone $\mathrm{H}$ (1h)

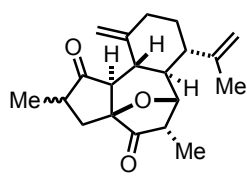

Curcusone I (1i: 2S) Curcusone $\mathrm{J}(1 \mathrm{j}$ : $2 R)$

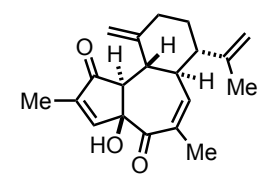

Curcusone $\mathrm{E}(\mathbf{1 e})$

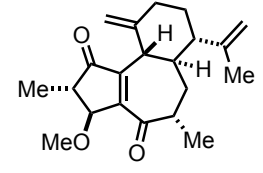

Curcusone F (1f)

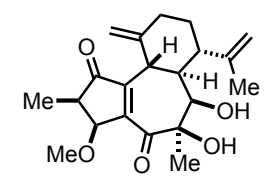

Curcusone $\mathrm{G}(\mathbf{1 g})$

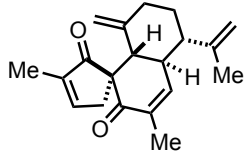

Spirocurcasone (3)

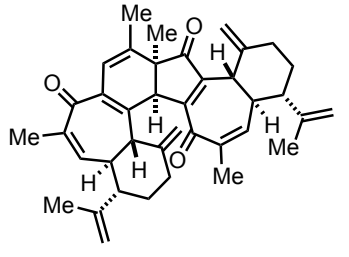

Dimericursone A (2a)

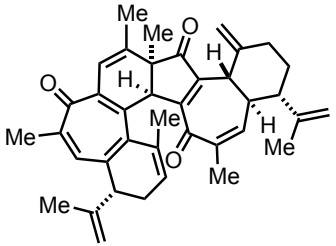

Dimericursone B (2b)

B Our Previous Total Syntheses of Curcusones I and J

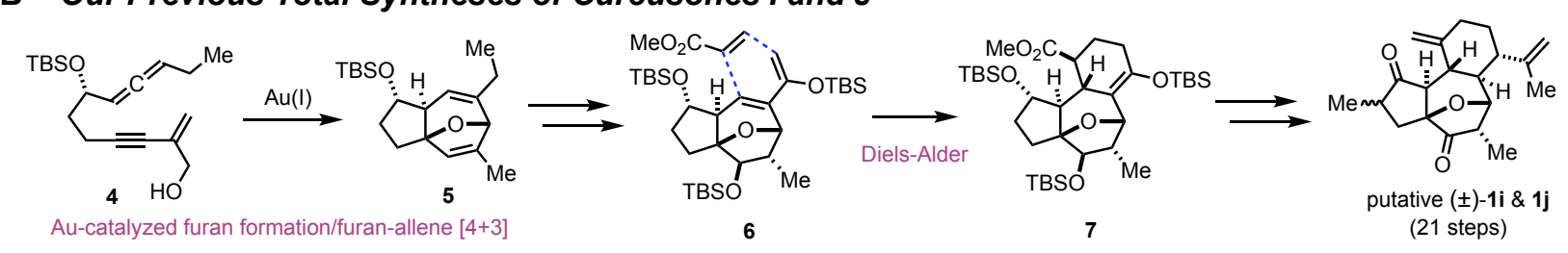

C Synthetic Studies toward the Curcusones by Stoltz et al.

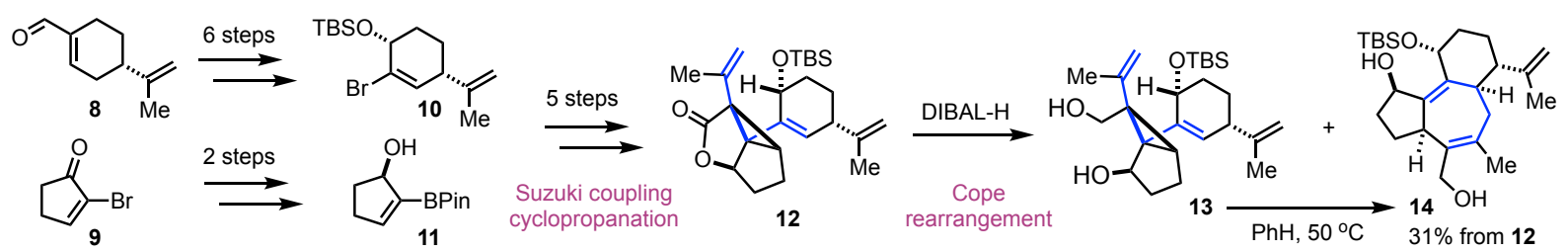

D Retrosynthetic Analysis

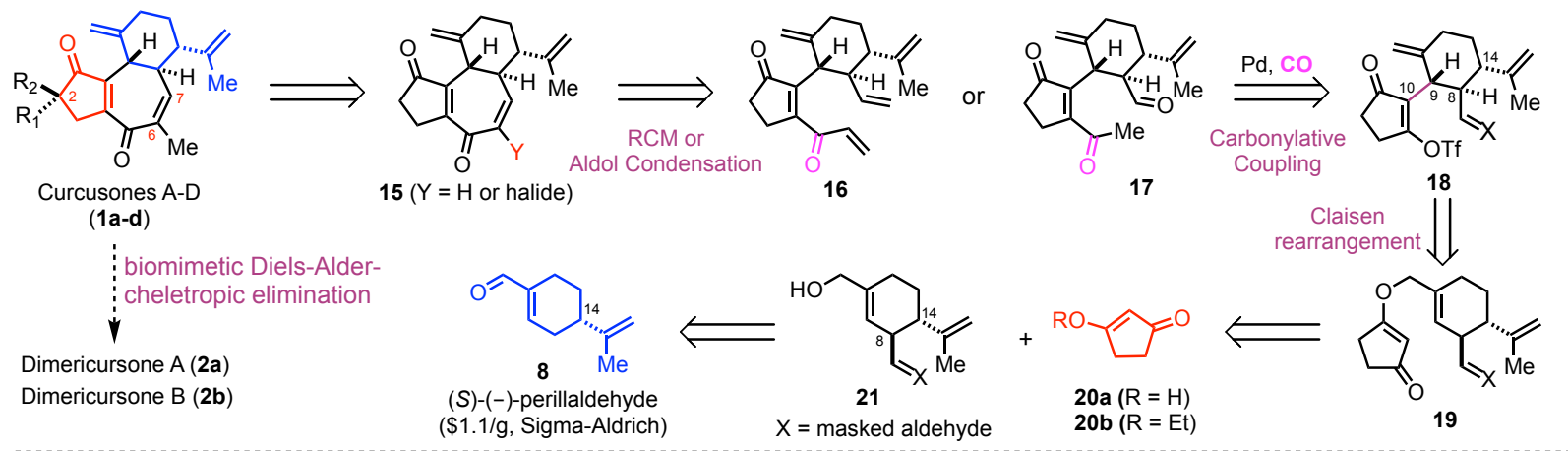

Fig. 1. Curcusone Diterpenes. (A) Selected curcusone diterpenes and their analogs. (B) Our total synthesis of the putative structures of curcusones I and J. (C) Synthetic efforts toward the curcusones from the Stotlz group. (D) Our retrosynthetic analysis of curcusones A-D. 
While the closely related daphnane and tigliane diterpenes have attracted a significant amount of synthetic interest ${ }^{9-13}$, the curcusone molecules have surprisingly received little attention despite their therapeutic potential. In 2017, we reported the first total syntheses of the putative structures of $\mathbf{1} \mathbf{i}$ and $\mathbf{1} \mathbf{j}$ in 21 steps (Fig. 1B), ultimately leading to the conclusion that the originally proposed structures of both $\mathbf{1 i}$ and $\mathbf{1} \mathbf{j}$ were incorrect ${ }^{14}$. Our synthesis involves a gold-catalyzed tandem furan formation and furan-allene [4+3] cycloaddition to build the 5,7-fused ring system with an oxa bridge and a Diels-Alder reaction to construct the 6-membered ring. In 2019, Stoltz and co-workers reported their studies toward synthesizing 1a-1d (Fig. 1C) ${ }^{15-17}$. Their approach features a divinylcyclopropane-cycloheptadiene rearrangement to forge the 7-membered ring and reached advanced intermediate $\mathbf{1 4}$ after 12 steps from $\mathbf{8 .}$

Total Synthesis and Probe Synthesis. Our ongoing interest in natural products that can covalently modify cellular proteins ${ }^{18}$ prompted us to continue pursuing the total synthesis and target identification of 1a-1d with an electrophilic cycloheptadienone moiety. This unique structural feature could allow them to form a covalent bond with nucleophilic residues of certain cellular proteins ${ }^{19}$. Previous cytotoxicity studies found that reduction and/or oxidation of the C6-C7 double bond greatly reduced their anticancer activity ${ }^{6}$. As such, an approach allowing variation of the C6 and C7 substituents would be highly desirable. We envisioned 15 as an advanced intermediate (Fig. 1D). $\alpha$-Halogenation followed by two methylation reactions would lead to $\mathbf{1 a}$ and $\mathbf{1 b}$, which could be oxidized to $\mathbf{1 c}$ and $\mathbf{1 d}$ via $\alpha$-hydroxylation. A ring closing metathesis $(\mathrm{RCM})$ or an intramolecular aldol condensation was planned to form the 7-membered dienone $(\mathbf{1 6} / \mathbf{1 7} \rightarrow \mathbf{1 5})$. Both 16 and 17 could be prepared from vinyl triflate 18 via Pd-catalyzed carbonylative cross couplings. At this stage, disconnection of the C9-C10 bond could break $\mathbf{1 8}$ into two simple pieces, but a direct intermolecular $\mathrm{C}-\mathrm{C}$ bond formation to construct such a bond is challenging. Thus, we opted for a Claisen rearrangement to forge this $\mathrm{C}-\mathrm{C}$ bond in a stereoselective manner and designed $\mathbf{1 9}$ with a masked aldehyde as the Claisen rearrangement precursor. 19 could be assembled from simple building blocks 20a/20b and 21. The latter could be derived from a cheap chiral pool molecule $(S)-(-)$-perillaldehyde $\mathbf{8}$. 

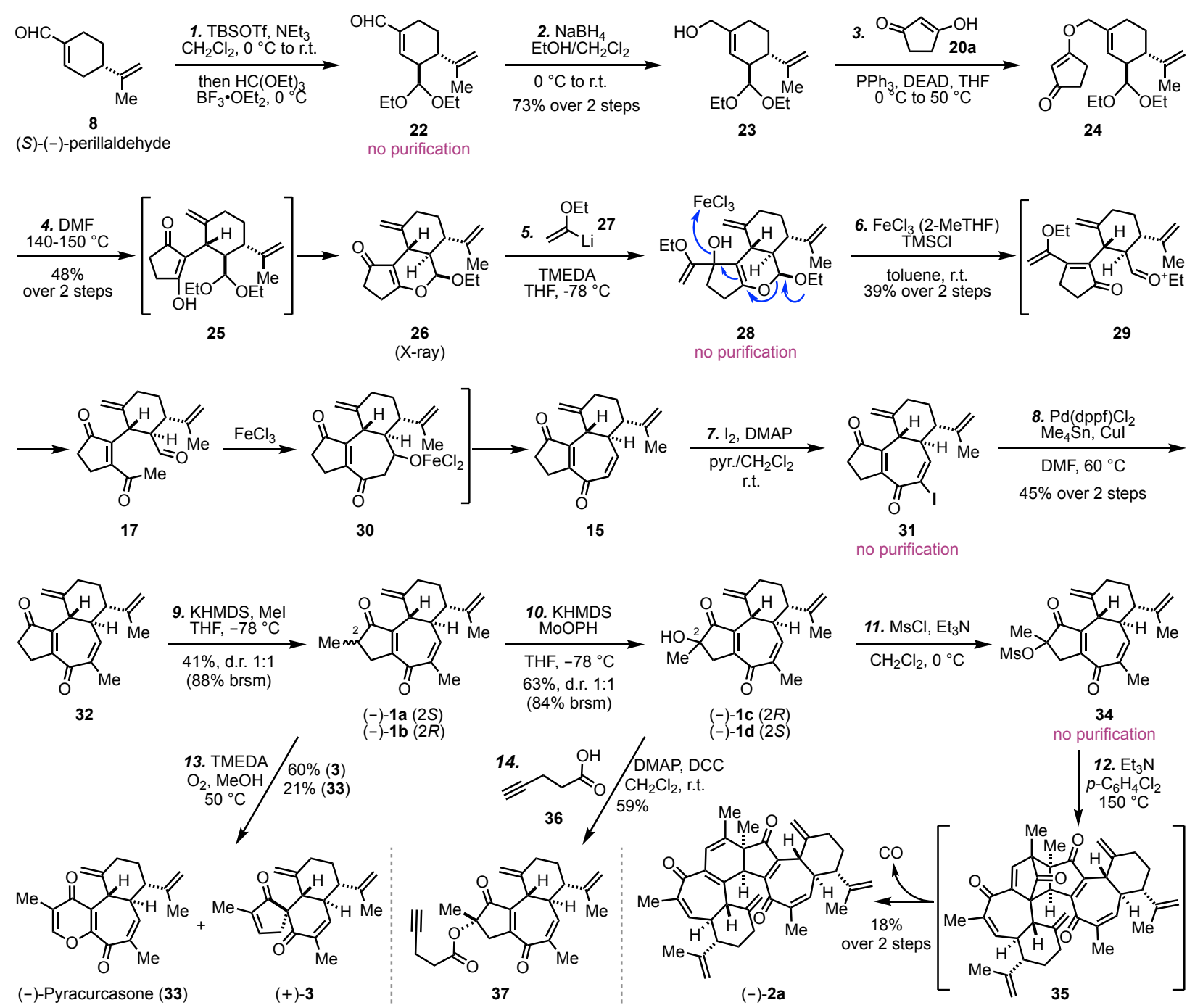

Fig. 2. Total syntheses of curcusones A-D and analogs. TBSOTf, tert-butyldimethylsilyl trifluoromethanesulfonate; DEAD, diethyl azodicarboxylate; DMF, dimethylformamide; TMEDA, $N, N, N^{\prime}, N^{\prime}$-tetramethylethylenediamine; 2-MeTHF， 2-methyltetrahydrofuran; TMSCl, trimethylsilyl chloride; DMAP, $\quad N, N$-dimethylaminopyridine; $\left.\quad \operatorname{Pd}(\operatorname{dppf}) \mathrm{Cl}_{2}\right), \quad\left[1,1^{\prime}\right.$-Bis(diphenylphosphino)ferrocene]dichloropalladium(II); KHMDS, potassium hexamethyldisilazide; MoOPH, oxodiperoxymolybdenum(pyridine)-(hexamethylphosphoric triamide); $\mathrm{MsCl}$, methanesulfonyl chloride; DCC, $N, N^{\prime}$-dicyclohexylcarbodiimide; r.t., room temperature $\left(23{ }^{\circ} \mathrm{C}\right)$; d.r., diastereomeric ratio; brsm, based on recovered starting material. 
Our synthesis started with preparing 23 (Fig. 2), a known compound synthesized from $\mathbf{8}$ in three steps - extended silyl enol ether formation, vinylogous Mukaiyama aldol reaction, and $\mathrm{NaBH}_{4}$ reduction ${ }^{20}$. We combined the first two steps into a one-pot reaction; crude 22 was then subjected to $\mathrm{NaBH}_{4}$ reduction directly to produce multi-decagram scale of $\mathbf{2 3}$ in one batch. We next needed to prepare $\mathbf{2 4}$ for the Claisen rearrangement. $\mathrm{NaH}$-promoted addition-elimination between $\mathbf{2 3}$ and $\mathbf{2 0 b}$ afforded 24, albeit in low yield (35\%). We then used a Mitsunobu reaction between 23 and 20a to prepare 24, but the hydrazine byproduct derived from diethyl azodicarboxylate could not be separated from $\mathbf{2 4}$. The recently reported redox-neutral organocatalytic Mitsunobu conditions were also explored but failed to provide $\mathbf{2 4}{ }^{21}$. Fortunately, the hydrazine byproduct could be tolerated in the Claisen rearrangement. After 24 was heated at $140-150{ }^{\circ} \mathrm{C}$ in DMF for $18 \mathrm{~h}$, the Claisen rearrangement did occur, but the rearranged product 25 further cyclized to provide tricyclic compound $\mathbf{2 6}$ (X-ray, Fig. S2) as a single diastereomer in 48\% yield from $\mathbf{2 3}$.

Without getting $\mathbf{2 5}$ to prepare triflate 18, we decided to continue with $\mathbf{2 6}$ and explore the hidden cyclopentane-1,3-dione symmetry to synthesize $\mathbf{1 7}$. We started with investigating 1,2-addition of lithiated ethyl vinyl ether (27) to $\mathbf{2 6}$ theorizing that a global hydrolysis would release the methyl ketone and the aldehyde at once to form 17 for the aldol condensation. This 1,2-addition turned out to be nontrivial. When two equiv. of $\mathbf{2 7}$ was used, only less than $10 \%$ yield of $\mathbf{2 8}$ was obtained. Owing to their oxophilicity, cerium chloride and lanthanum chloride have been used to promote 1,2-additions ${ }^{22}$. Unfortunately, both failed in our case. Eventually, the 1,2-addition was improved by increasing the amount of $\mathbf{2 7}$ to 10 equiv. ${ }^{23}$, and $\mathbf{2 8}$ was prepared in 57\% yield. 28 was then subjected to hydrolysis upon the treatment with $p$-toluenesulfonic acid and $\mathbf{1 7}$ was obtained in 51\% yield from 26. Meanwhile, we were delight to observe the formation of 15 in the same reaction, albeit in very poor yield $(<5 \%)$. We were encouraged to achieve a global hydrolysis/aldol condensation cascade to synthesize $\mathbf{1 5}$ from $\mathbf{2 8}$ in one step and identified $\mathrm{FeCl}_{3}{ }^{24}$ in combination with $\mathrm{TMSCl}$ as the optimal conditions. When crude 28 was treated with a premixed $\mathrm{FeCl}_{3}$ (0.2 $\mathrm{M}$ in 2-methyltetrahydrofuran) and $\mathrm{TMSCl}$ in toluene at room temperature, global hydrolysis occurred to generate 17 in situ, which further underwent $\mathrm{FeCl}_{3}$-promoted intramolecular aldol condensation to afford $\mathbf{1 5}$ in 39\% yield from $\mathbf{2 6}$. 
With the [6-7-5] tricyclic carbon skeleton quickly assembled in only six steps, we next needed to introduce the two methyl groups. Johnson iodination converted 15 to iodoenone 31, which was surprisingly unstable. Therefore, after a quick workup, crude $\mathbf{3 1}$ was immediately subjected to the next Stille cross coupling with tetramethylstannane to provide 32 in $45 \%$ yield over two steps. Finally, $\alpha$-methylation of enone 32 at C2 position delivered a 1:1 mixture of separable (-)-1a and (-)-1b in 41\% yield (88\% brsm; 9 steps total). $\alpha$-Hydroxylation of 1a with KHDMS and MoOPH gave separable (-)-1c and (-)-1d in 63\% yield (d.r. 1:1; $84 \%$ brsm; 10 steps total). Additionally, in order to obtain analogs for biological activity comparison, we converted (-)-1b to (+)-3 and (-)-33 (a synthetic derivative named as pyracurcasone) by following a reported one-step procedure 7 . The ${ }^{1} \mathrm{H},{ }^{13} \mathrm{C}$ NMR, and other analytic data of our synthetic samples matched well with the reported ones, which also conclude that the absolute configuration of 1a-1d assigned by Clardy et al. in 1986 is opposite of the actual ones.

We then set out to synthesize 2a from 1a-1d via a biomimetic dimerization. The proposed biosynthesis of $\mathbf{2 a}$ consists of a sequence of oxidative dehydrogenation of $\mathbf{1 a} / \mathbf{1 b}$ or dehydration of $\mathbf{1} / \mathbf{1} \mathbf{d}$ to form a reactive cyclopentadienone intermediate followed by Diels-Alder dimerization and cheletropic extrusion of carbon monoxide ${ }^{8}$. From there, $\mathbf{2 a}$ could be converted to $\mathbf{2} \mathbf{b}$ via another oxidative dehydrogenation and double bond isomerization. We started with 1c and 1d. After an unfruitful attempt to synthesize 2a by heating them directly at elevated temperatures, a 1:1 mixture of them was first converted to their mesylates (34). After extensive exploration, we identified that using triethylamine as a base in 1,4dichlorobenzene at $150{ }^{\circ} \mathrm{C}$ produced (-)-2a in $18 \%$ yield over two steps, which provides a direct evidence to support the proposed biosynthetic pathway. Under our conditions, the formation of $\mathbf{2 b}$ was not observed.

To elucidate the anticancer mechanism and identify potential cellular targets of curcusones, an alkyne-tagged probe molecule $\mathbf{3 7}$ was designed for chemoproteomics studies. Since the dienone is likely protein-reactive and is critical for the observed activity, we decided to minimize structural perturbation of this part and used the tertiary alcohol as a handle to link with a terminal alkyne. $\mathbf{3 7}$ was synthesized in 59\% yield from (-)-1d via a DCC-promoted coupling with $\mathbf{3 6}$. 
Cytotoxicity and Target Identification. We evaluated the cytotoxicity of curcusones and their analogs in breast cancer MCF-7 cells using the WST-1 assay (Fig. 3A). Synthetic 1a-1d, natural 1b and 1d, and intermediates $\mathbf{1 5}$ and $\mathbf{3 2}$ exhibited micromolar $\mathrm{EC}_{50}$ values against MCF-7 cells with $\mathbf{1 d}$ being the most potent curcusone. Importantly, the cytotoxicity values for synthetic $\mathbf{1 b}$ and $\mathbf{1 d}$ were virtually identical to the values of their naturally isolated counterparts. Analog 33 showed slightly better antiproliferation activity indicating the feasibility of finely tuning the cycloheptadienone moiety to improve potency, but 2a was not active even at $100 \mu \mathrm{M}$. Likely due to the full confluency of the tested MCF-7 cells, the $\mathrm{EC}_{50}$ values we obtained were about one order of magnitude higher than previously reported (1.6-3.1 $\mu \mathrm{M} \mathrm{EC}_{50}$ values for 1a-1d) ${ }^{6}$. Gratifyingly, 37 retained similar anticancer properties of 1d, thus warranting its use in competitive chemoproteomic studies.

We then identified the cellular targets of curcusones by competitive chemoproteomics using probe 37. MCF-7 cells were treated with 1d for 4 hours followed by lysis, treatment with 37, CuAAC with biotin azide, enrichment, digestion, and LC-MS/MS analysis using label-free quantification (Fig. 3B and Table $\mathrm{S} 1)$. The best competed target was BRAT1, which acts as a master regulator of the DNA damage response (DDR) and DNA repair by binding to BRCA1 and by activating DDR kinases such as ATM and PRKDC (DNA-PKcs) following DNA damage ${ }^{25-27}$. Knockdown of BRAT1 increased the constitutive level of apoptosis in human osteosarcoma cells ${ }^{25}$ and decreased cancer cell proliferation and tumorigenicity in vitro and in mouse tumor xenografts ${ }^{27}$. BRAT1 is also an unfavorable prognostic marker in kidney and liver cancers $^{28}$. Therefore, targeting BRAT1 is a promising strategy for cancer treatment. 


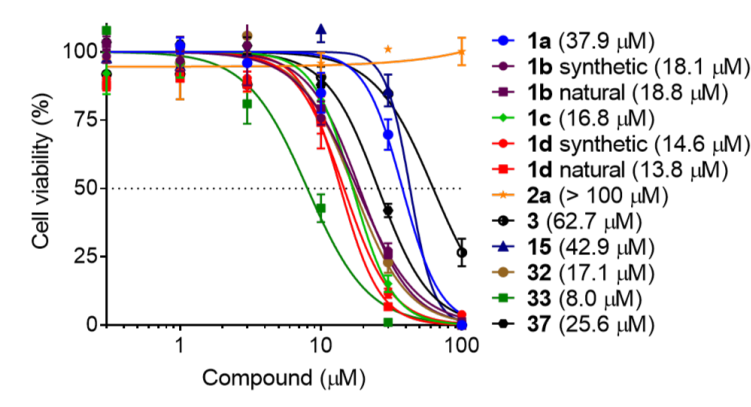

C

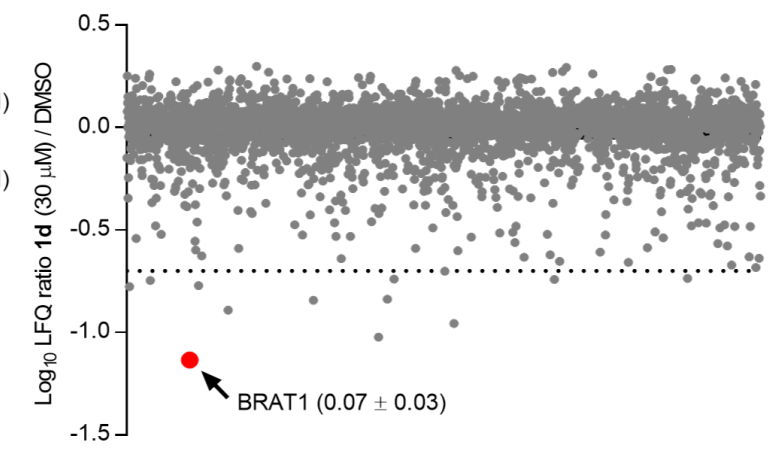

D
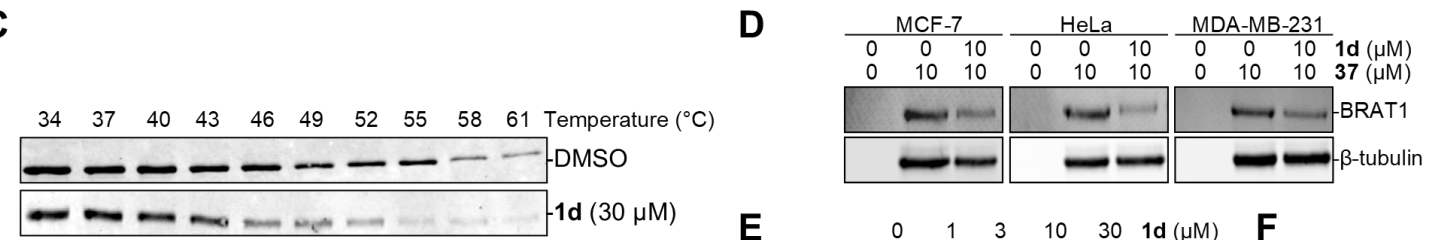

E

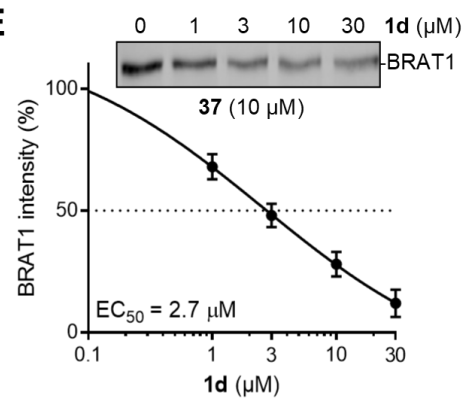

$\mathbf{F}$
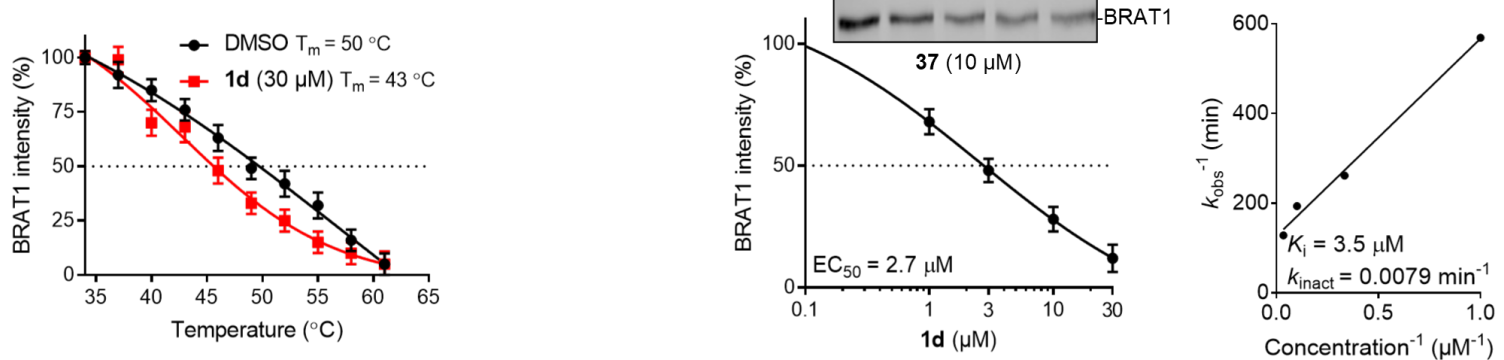

Fig. 3. Identification of BRAT1 as a cellular target of 1d. (A) Viability of MCF-7 cells following $24 \mathrm{~h}$ compound treatment with $\mathrm{EC}_{50}$ values in brackets $(n=3)$. (B) Scatter plot of proteins competed by $\mathbf{1 d}$ from probe $37(10 \mu \mathrm{M})$ enrichment in MCF-7 cells with BRAT1 highlighted $(n=3)$. (C) Western blot (top) and quantification (bottom) of thermal shift assay of overexpressed FLAG-BRAT1 lysate treated with 1d. (D) Western blots of BRAT1 following treatment of live cells with 1d and enrichment by $\mathbf{3 7}$ in competitive pulldown assays. (E) Western blot (top) and quantification (bottom) of BRAT1 in a dose-dependent competitive pulldown assay in HeLa cells. (F) Determination of the binding constants $K_{\mathrm{i}}$ and $k_{\text {inact }}$ for $\mathbf{1 d}$ against BRAT1 using a competitive pulldown assay. All error bars are S.D.

We next characterized the physical interaction between 1d and BRAT1. We overexpressed FLAGBRAT1 in HEK-293T cells and performed a thermal shift assay by treating lysates with 1d, heating as indicated, and probing the remaining soluble FLAG-BRAT1 by Western blotting (Fig. 3C). We observed 
thermal destabilization of 1d-treated BRAT1 indicating a direct interaction. To validate endogenous BRAT1 as a target of $\mathbf{1 d}$ in live cells, we employed a competitive pulldown experiment. MCF-7 cells were treated with $1 \mathbf{d}$ for 4 hours before lysis, treatment with probe 37, CuAAC with biotin azide, streptavidin enrichment, elution, and Western blot visualization. Indeed, native BRAT1 was enriched by 37 and was competed by 1d (Fig. 3D). Additionally, 1d competed the enrichment of BRAT1 from cervical cancer HeLa and triple negative breast cancer MDA-MB-231 cells, thus validating native BRAT1 as a cellular target of $\mathbf{1 d}$ across these cell lines. In situ treatment of $\mathbf{1 d}$ in live HeLa cells competed BRAT1 enrichment by $\mathbf{3 7}$ at low micromolar concentrations $\left(\mathrm{EC}_{50}=2.7 \mu \mathrm{M}\right.$; Fig. 3E). Assuming that 1d binds to BRAT1 irreversibly, we determined the binding constants $K_{\mathrm{i}}(3.5 \mu \mathrm{M})$ and $k_{\text {inact }}\left(0.0079 \mathrm{~min}^{-1}\right.$; Fig. 3F). These results demonstrate that $\mathbf{1 d}$ is the first small-molecule binder of BRAT1.

BRAT1 Modulation. To determine whether 1d inhibits BRAT1 in cells, we generated stable BRAT1 KD HeLa cells via shRNA retroviral transduction (Fig. S1A). We then compared the protein expression profiles of BRAT1 KD cells versus 1d-treated cells $(3 \mu \mathrm{M}, 24 \mathrm{~h})$ by global proteomics analysis (Fig. 4A-C and Table S2). Among 3347 quantified proteins in compound-treated cells, we found only 36 up- and 42 down-regulated proteins. Importantly, 31 of the 78 dysregulated proteins were also dysregulated in BRAT1 KD cells, thus indicating that 1d functionally inhibits BRAT1 in cells. Notably, several wellknown cancer migration and progression drivers were downregulated (Fig. 4B), including TRIM47 which mediates cancer migration ${ }^{29}$, the bona fide oncoprotein and potential biomarker $\mathrm{WBP}^{30}$, and frequently highly amplified oncogene FNDC $3 \mathrm{~B}^{31}$. None of these proteins have previously been functionally linked to BRAT1. We then investigated the effect of $\mathbf{1 d}$ treatment and BRAT1 KD on cancer cell migration in WT and BRAT1 KD HeLa cells, as well as WT MCF-7 and MDA-MB-231 cells (Fig. 4D-G). As expected, BRAT1 knockdown greatly diminished migration of HeLa cells, and treatment with 1d at $1 \mu \mathrm{M}$ concentration also reduced migration of all cell lines by $\sim 4$-fold. 
A

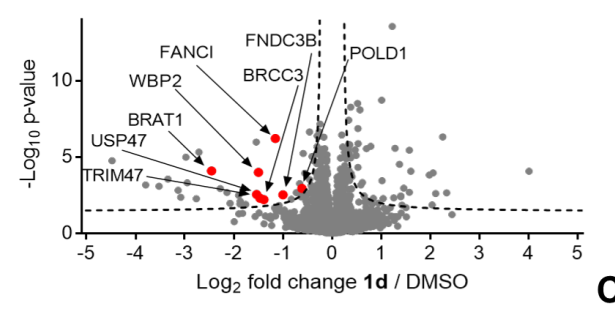

B

C

\begin{tabular}{|l|c|c|c|}
\hline \multicolumn{1}{|c|}{ Protein name } & Gene name & $\begin{array}{c}\text { Ratio 1d/ } \\
\text { DMSO }\end{array}$ & $\begin{array}{c}\text { Ratio } \\
\text { KD/WT }\end{array}$ \\
\hline Fibronectin type III domain-containing protein 3B & FNDC3B & 0.50 & 0.56 \\
\hline Tripartite motif-containing protein 47 & TRIM47 & 0.38 & 0.44 \\
\hline WW domain-binding protein 2 & WBP2 & 0.35 & 0.44 \\
\hline
\end{tabular}

\begin{tabular}{|l|c|c|c|}
\hline \multicolumn{1}{|c|}{ Protein name } & Gene name & $\begin{array}{c}\text { Ratio 1d/ } \\
\text { DMSO }\end{array}$ & $\begin{array}{c}\text { Ratio } \\
\text { KD/WT }\end{array}$ \\
\hline Lys-63-specific deubiquitinase BRCC36 & BRCC3 & 0.34 & 0.54 \\
\hline Fanconi anemia group I protein & FANCI & 0.45 & 0.56 \\
\hline DNA polymerase delta catalytic subunit & POLD1 & 0.65 & 0.38 \\
\hline Ubiquitin carboxyl-terminal hydrolase 47 & USP47 & 0.36 & 0.46 \\
\hline
\end{tabular}

D

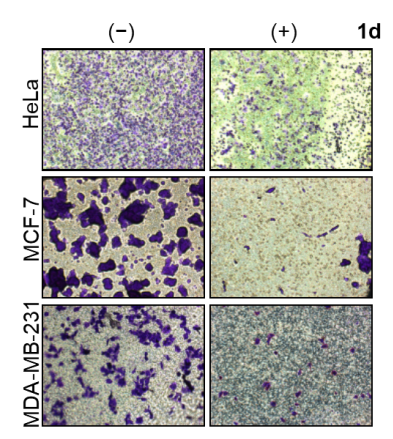

E

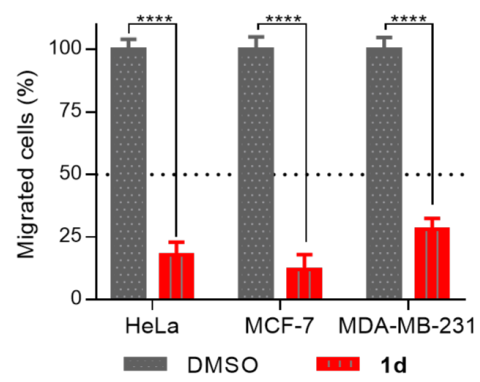

F

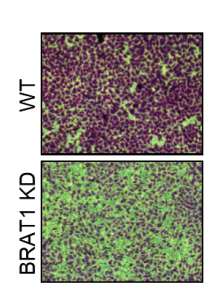

G

H

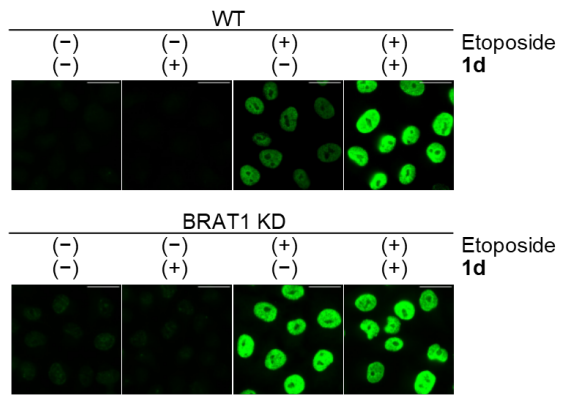

J
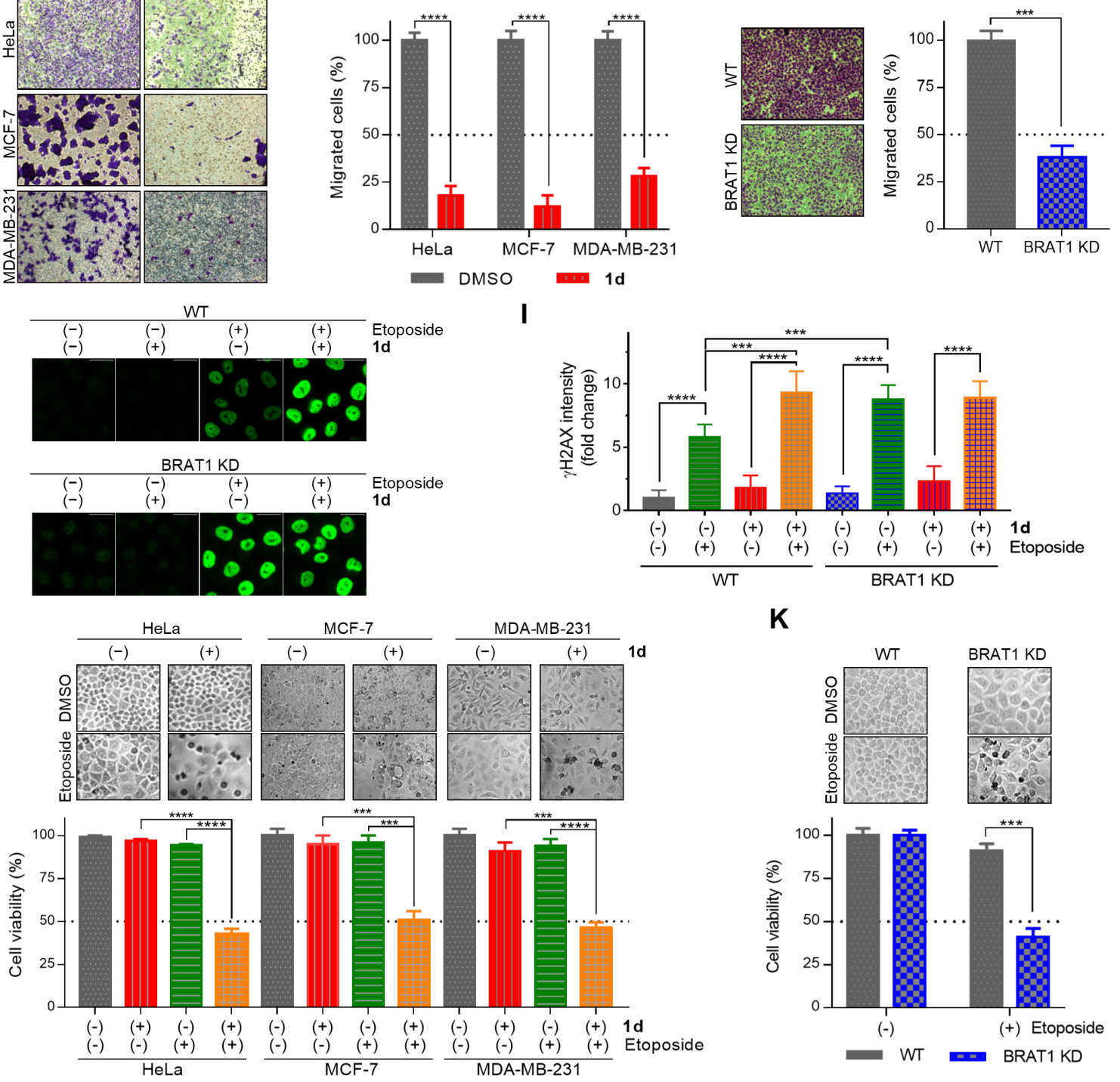

K
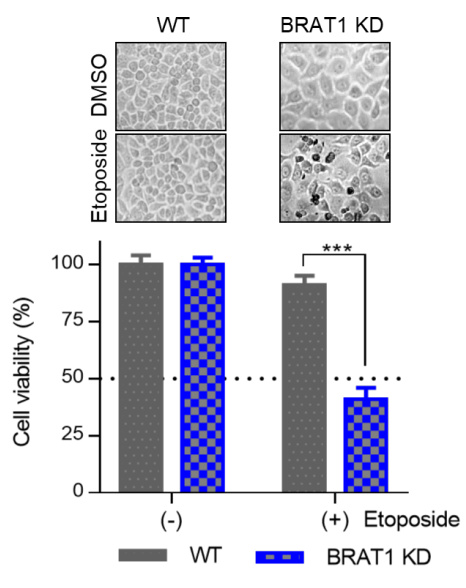
Fig. 4. Evaluation of BRAT1 inactivation by 1d. (A) Global proteomic analysis of HeLa cells comparing 1d treatment to BRAT1 knockdown versus DMSO or non-targeting (WT) controls, respectively. Color dots indicate proteins which are downregulated in both conditions $(n=3)$. (B) Table showing common downregulated proteins involved in cell migration and, (C) in DNA repair. (D) Crystal violet images and, (E) quantification of cells in a transwell migration assay following $1 \mathbf{d}(1 \mu \mathrm{M} ; 24 \mathrm{~h})$ treatment $(n=3)$.

Crystal violet images and, $(\mathbf{G})$ quantification of WT and BRAT1 KD cells (24 h) in a transwell migration assay $(n=3)$. (H) Fluorescence microscopy images and, (I) quantification of WT or BRAT1 KD cells treated with DMSO, 1d $(3 \mu \mathrm{M})$, etoposide $(30 \mu \mathrm{M})$, or both compounds showing $\gamma \mathrm{H} 2 \mathrm{AX}$ staining $(n=3)$. (J) Images of cells (top) and quantification (bottom) after $24 \mathrm{~h}$ treatment with DMSO, 1d (3 $\mu \mathrm{M})$, etoposide $(50 \mu \mathrm{M})$, or both compounds or, $(\mathbf{K})$ following BRAT1 knockdown and subsequent treatment with etoposide. All error bars are S.D. All scale bars indicate $30 \mu \mathrm{m} . * * * P<0.001, * * * * P<0.0001$, Welch's twosided t-test.

Our global proteomics experiment also revealed several commonly downregulated key DNA repair proteins (Fig. 4C) such as (i) POLD1 which synthesizes DNA during repair ${ }^{32}$, (ii) USP47 which facilitates base-excision repair ${ }^{33}$, (iii) FANCI which mediates the repair of DNA double strand breaks and interstrand $\operatorname{crosslinks}^{34}$, and (iv) BRCC 3 which stabilizes the accumulation of BRCA1 at DNA breaks ${ }^{35}$. These proteins have not been previously linked to BRAT1 either. Most notably, 1d treatment (24 hours) significantly downregulated the actual physical target, BRAT1 (ratio 0.18), as confirmed by Western blotting (Fig. S1B). Collectively, these findings demonstrate the importance of BRAT1 as a master regulator of the DDR and that 1d inhibits BRAT1 in cells.

We then investigated whether 1d would potentiate the DNA damaging effect of the clinical drug and topoisomerase inhibitor etoposide via BRAT1 inhibition. WT or BRAT1 KD HeLa cells were treated with DMSO, etoposide, 1d, or etoposide and 1d combined. Subsequent DNA damage was then measured by fluorescence microscopy using $\gamma \mathrm{H} 2 \mathrm{AX}$ staining (Fig. 4H, I and Fig. S1C). Treatment with $\mathbf{1 d}(3 \mu \mathrm{M})$ or KD of BRAT1 alone did not increase $\gamma \mathrm{H} 2 \mathrm{AX}$ signal. However, co-treatment of $\mathbf{1 d}$ with etoposide led to a 
2-fold increase. Similarly, etoposide treatment significantly increased $\gamma \mathrm{H} 2 \mathrm{AX}$ signal in BRAT1 KD cells, recapitulating the 1d/etoposide co-treatment results. Importantly, 1d treatment did not increase $\gamma \mathrm{H} 2 \mathrm{AX}$ signal in etoposide-treated BRAT1 KD cells, confirming that the 1d-etoposide synergism is linked to BRAT1 inactivation. Furthermore, co-treatment of $1 \mathbf{d}$ with etoposide also increased cytotoxicity in HeLa, MCF-7, and MDA-MB-231 cells (Fig. 4J). Likewise, there was increased cell death in BRAT1 KD HeLa cells following etoposide treatment relative to WT cells (Fig. 4K). Altogether, these results demonstrate that targeting BRAT1 with 1d is a promising anticancer strategy for chemosensitization to DNA damaging drugs.

In summary, we completed the first asymmetric total synthesis and target identification of the curcusone natural products. Our convergent synthesis builds upon a cheap and abundant chiral pool molecule (8) and features a thermal [3,3]-sigmatropic rearrangement and an $\mathrm{FeCl}_{3}$-promoted global hydrolysis/aldol condensation cascade to rapidly construct the critical cycloheptadienone core. This efficient synthetic route yielded 1a and 1b in 9 steps, 1c and 1d in 10 steps, and 2a in 12 steps from $(S)$ (-)-8. The successful synthesis of 2a from 1c/1d experimentally supports the proposed Diels-Alder dimerization and cheletropic extrusion biosynthesis. By performing chemoproteomics with the alkyne probe 37, we identified the previously "undruggable" oncogenic protein BRAT1 as a key cellular target of 1d. Furthermore, 1d inhibits BRAT1 in cancer cells, thereby reducing cancer cell migration, increasing susceptibility to DNA damage, and inducing chemosensitization to the approved drug etoposide. To our knowledge, 1d is the first known small-molecule inhibitor of BRAT1, a master regulator of the DDR and DNA repair. Many promising clinical trials are underway targeting DDR proteins such as PARP, ATR, ATM, CHK, and DNA-PK as monotherapies or in combination with other treatments ${ }^{36-37}$. Olaparib, a PARP inhibitor, was approved by FDA in 2014 as a monotherapy to treat germline BRCA1/2-mutant ovarian cancer $^{36}$. Further structure-activity optimizations of the curcusones may thus yield novel BRTAT1 inhibitors as potential lead medicines for monotherapies or combination therapies.

\section{Experimental Procedures and Data}


For detailed experimental procedures and compound characterization data, see the Supplementary Information.

\section{Acknowledgements}

We thank Dr. Dany Pechalrieu for helpful discussions. Financial support is provided by the NIH (R35 GM128570), the NSF Graduate Research Fellowships Program, the Reba and Nat Newman Endowed Fellowship, and The Scripps Research Institute. The NIH P30CA023168 is supporting shared NMR resources to Purdue Center for Cancer Research.

\section{Competing financial interests}

M.D., A.A., C.C., B.G.D., Z.C. are inventors on patent application U.S. 63/084,594 submitted by Purdue University that covers the synthesis of curcusone derivatives.

Correspondence and requests for materials should be addressed to M.D. and A.A.

\section{References:}

1. Harvey, A. L., Edrada-Ebel, R. \& Quinn, R. J. The re-emergence of natural products for drug discovery in the genomics era. Nat. Rev. Drug Discov. 14, 111-129 (2015).

2. Nicolaou, K. C. Advancing the drug discovery and development process. Angew. Chem. Int. Ed. 53, 9128-9140 (2014).

3. Dang, C. V., Reddy, E. P., Shokat, K. M. \& Soucek, L. Drugging the 'undruggable' cancer targets. Nat. Rev. Cancer 17, 502-508 (2017).

4. Wang, H. B., Wang, X. Y., Liu, L. P., Qin, G. W. \& Kang, T. G. Tigliane diterpenoids from the Euphorbiaceae and Thymelaeaceae families. Chem. Rev. 115, 2975-3011 (2015).

5. Naengchomnong, W., Thebtaranonth, Y., Wiriyachitra, P., Okamoto, K. T. \& Clardy, J. Isolation and structure determination of four novel diterpenes from jatropha curcus. Tetrahedron Lett. 27, 2439-2442 (1986). 
6. Liu, J.-Q., Yang, Y.-F., Li, X.-Y., Liu, E.-Q., Li, Z.-R., Zhou, L., Li, Y. \& Qiu, M.-H. Cytotoxicity of naturally occurring rhamnofolane diterpenes from Jatropha curcas. Phytochemistry 96, 265-272 (2013).

7. Li, X.-Y., Yang, Y.-F., Peng, X.-R., Li, M.-M., Li, L.-Q., Deng, X., Qin, H.-B., Liu, J.-Q. \& Qiu, M.H. One-step semisynthesis method of spirocurcasone and pyracurcasone from curcusones A and B. Org. Lett. 16, 2196-2199 (2014).

8. Liu, J.-Q., Xu, Y., Xiao, Q., Huang, J.-D., Ma, J.-J., Lian, C.-L., Huang, M.-Y., Du, Z. \& Wang, C.-F. Dimericursones A and B: two unprecedented hexacyclic dimeric diterpenoids from the root barks of Jatropha curcas. Org. Biomol. Chem. 16, 8305-8310 (2018).

9. Wender, P. A., Jesudason, C. D., Nakahira, H., Tamura, N., Tebbe, A. L. \& Ueno, Y. The first synthesis of a daphnane diterpene: The enantiocontrolled total synthesis of (+)-resiniferatoxin. J. Am. Chem. Soc. 119, 12976-12977 (1997).

10. Hashimoto, S., Katoh, S. I., Kato, T., Urabe, D. \& Inoue, M. Total Synthesis of Resiniferatoxin Enabled by Radical-Mediated Three-Component Coupling and 7-endo Cyclization. J. Am. Chem. Soc. 139, 16420-16429 (2017).

11. Wender, P. A., Rice, K. D. \& Schnute, M. E. The first formal asymmetric synthesis of phorbol. J. Am. Chem. Soc. 119, 7897-7898 (1997).

12. Kawamura, S., Chu, H., Felding, J. \& Baran, P. S. Nineteen-step total synthesis of (+)-phorbol. Nature 532, 90-93 (2016).

13. Wender, P. A., Kee, J. M. \& Warrington, J. M. Practical synthesis of prostratin, DPP, and their analogs, adjuvant leads against latent HIV. Science 320, 649-652 (2008).

14. Li, Y. \& Dai, M. Total Syntheses of the Reported Structures of Curcusones I and J through Tandem Gold Catalysis. Angew. Chem. Int. Ed. 56, 11624-11627 (2017).

15. Lee, C. W., Taylor, B. L. H., Petrova, G. P., Patel, A., Morokuma, K., Houk, K. N. \& Stoltz, B. M. An Unexpected Ireland-Claisen Rearrangement Cascade During the Synthesis of the Tricyclic Core of Curcusone C: Mechanistic Elucidation by Trial-and-Error and Automatic Artificial Force-Induced Reaction (AFIR) Computations. J. Am. Chem. Soc. 141, 6995-7004 (2019). 
16. Wright, A. C.; Lee, C. W. \& Stoltz, B. M. Progress toward the Enantioselective Synthesis of Curcusones A-D via a Divinylcyclopropane Rearrangement Strategy. Org. Lett. 21, 9658-9662 (2019).

17. Wright, A. C. \& Stoltz, B. M. Enantioselective construction of the tricyclic core of curcusones A-D via a cross-electrophile coupling approach. Chem. Sci. 10, 10562-10565 (2019).

18. Davis, D. C., Hoch, D. G., Wu, L., Abegg, D., Martin, B. S., Zhang, Z.-Y., Adibekian, A. \& Dai, M. Total Synthesis, Biological Evaluation, and Target Identification of Rare Abies Sesquiterpenoids. $J$. Am. Chem. Soc. 140, 17465-17473 (2018).

19. Jackson, P. A., Widen, J. C., Harki, D. A. \& Brummond, K. M. Covalent Modifiers: A Chemical Perspective on the Reactivity of $\alpha, \beta$-Unsaturated Carbonyls with Thiols via Hetero-Michael Addition Reactions. J. Med. Chem. 60, 839-885 (2017).

20. Abe, H., Sato, A., Kobayashi, T. \& Ito, H. Concise total synthesis of spirocurcasone. Org. Lett. 15, 1298-1301 (2013).

21. Beddoe, R. H., Andrews, K. G., Magne, V., Cuthbertson, J. D., Saska, J., Shannon-Little, A. L., Shanahan, S. E., Sneddon, H. F. \& Denton, R. M. Redox-neutral organocatalytic Mitsunobu reactions. Science 365, 910-914 (2019).

22. Krasovskiy, A., Kopp, F. \& Knochel, P. Soluble lanthanide salts $\left(\mathrm{LnCl}_{3} \cdot 2 \mathrm{LiCl}\right)$ for the improved addition of organomagnesium reagents to carbonyl compounds. Angew. Chem. Int. Ed. 45, 497-500 (2006).

23. Haelsig, K. T., Xuan, J. \& Maimone, T. J. Total Synthesis of (-)-Curvulamine. J. Am. Chem. Soc. 142, $1206-1210(2020)$.

24. Ludwig, J. R., Zimmerman, P. M., Gianino, J. B. \& Schindler, C. S. Iron(III)-catalyzed carbonyl-olefin metathesis. Nature 533, 374-370 (2016).

25. Aglipay, J. A., Martin, S. A., Tawara, H., Lee, S. W. \& Ouchi, T. ATM activation by ionizing radiation requires BRCA1-associated BAAT1. J. Biol. Chem. 281, 9710-9718 (2006).

26. So, E. Y. \& Ouchi, T. Functional interaction of BRCA1/ATM-associated BAAT1 with the DNA-PK catalytic subunit. Exp. Ther. Med. 2, 443-447 (2011). 
27. So, E. Y. \& Ouchi, T. BRAT1 deficiency causes increased glucose metabolism and mitochondrial malfunction. BMC Cancer 14, 548, (2014).

28. The Human Protein Atlas: BRAT1 Pathology, <https://www.proteinatlas.org/ENSG00000106009BRAT1/pathology> (2020).

29. Han, Y., Tian, H., Chen, P. \& Lin, Q. TRIM47 overexpression is a poor prognostic factor and contributes to carcinogenesis in non-small cell lung carcinoma. Oncotarget 8, 22730-22740, (2017).

30. Tabatabaeian, H., Rao, A., Ramos, A., Chu, T., Sudol, M. \& Lim, Y. P. Oncogene 39, 4621-4635 (2020).

31. Chen, C.-F., Hsu, E.-C., Lin, K.-T., Tu, P.-H., Chang, H.-W., Lin, C.-H., Chen, Y.-J., Gu, D.-L., Lin, C.-H., Wu, J.-Y., Chen, Y.-T., Hsu, M.-T. \& Jou, Y.-S. Overlapping high-resolution copy number alterations in cancer genomes identified putative cancer genes in hepatocellular carcinoma. Hepatology 52, 1690-1701 (2010).

32. Nicolas, E., Golemis, E. A. \& Arora, S. POLD1: Central mediator of DNA replication and repair, and implication in cancer and other pathologies. Gene 590, 128-141 (2016).

33. Nickson, C. M. \& Parsons, J. L. Monitoring regulation of DNA repair activities of cultured cells in-gel using the comet assay. Front. Genet. 5, 232 (2014).

34. Smogorzewska, A., Matsuoka, S., Vinciguerra, P., McDonald $3^{\text {rd }}$, E. R., Hurov, K. E., Luo, J., Ballif, B. A., Gygi, S. P., Hofmann, K., D’Andrea, A. D. \& Elledge, S. J. Identification of the FANCI protein, a monoubiquitinated FANCD2 paralog required for DNA repair. Cell 129, 289-301 (2007).

35. Dong, Y., Hakimi, M.-A., Chen, X., Kumaraswamy, E., Cooch, N. S., Godwin, A. K. \& Shiekhattar, R. Regulation of BRCC, a holoenzyme complex containing BRCA1 and BRCA2, by a signalosomelike subunit and its role in DNA repair. Mol. Cell 12, 1087-1099 (2003).

36. Pearl, L. H., Schierz, A. C., Ward, S. E., Al-Lazikani, B. \& Pearl, F. M. Therapeutic opportunities within the DNA damage response. Nat. Rev. Cancer 15, 166-180 (2015).

37. de Bono, J., Mateo, J., Fizazi, K., Saad, F., Shore, N., Sandhu, S., Chi, K. N., Sartor, O., Agarwal, N., Olmos, D., Thiery-Vuillemin, A., Twardowski, P., Mehra, N., Goessl, C., Kang, J., Burgents, J., Wu, 
W., Kohlmann, A., Adelman, C. A. \& Hussain, M. Olaparib for Metastatic Castration-Resistant Prostate Cancer. N. Engl. J. Med. 382, 2091-2102 (2020).

\section{Supplementary Figures}

A

$(-) \quad(+)$ BRAT1 ShRNA
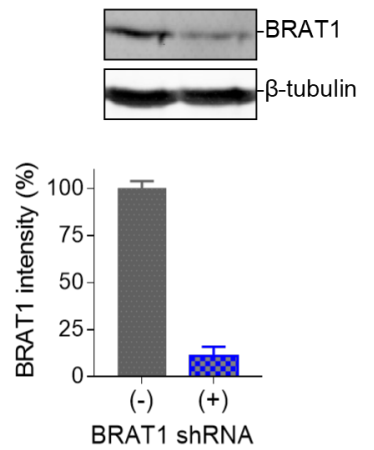

B
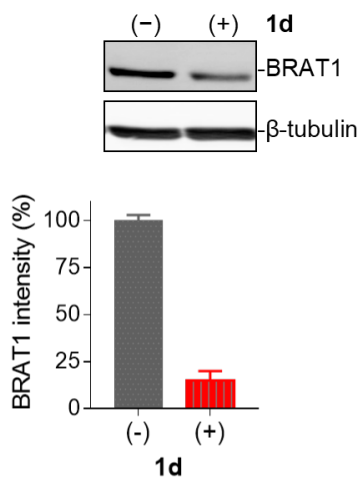

C

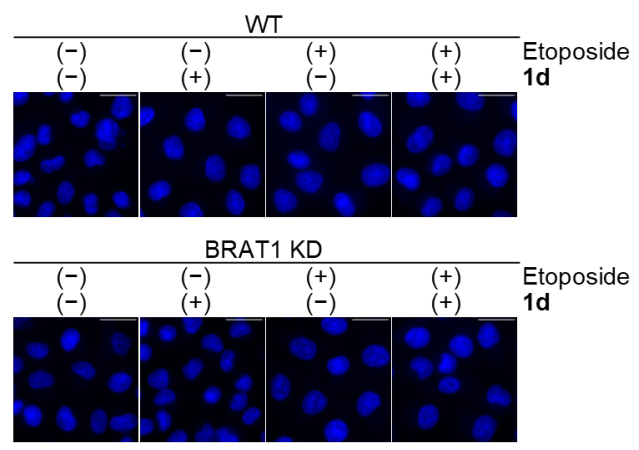

Fig. S1. (A) Western blot (top) and quantification (bottom) of BRAT1 in HeLa cells following shRNA retroviral transduction. (B) Western blot (top) and quantification (bottom) of BRAT1 in HeLa cells following treatment with $\mathbf{1 d}(3 \mu \mathrm{M})$ for 24 hours. (C) Fluorescence microscopy images of WT or BRAT1 KD cells treated with DMSO, 1d $(3 \mu \mathrm{M})$, etoposide $(30 \mu \mathrm{M})$, or both compounds showing DAPI staining. All error bars are S.D. 